\title{
Estudios Cinéticos de la Disolución de Hidroboracita en Soluciones de Acido Clorhídrico y Acido Sulfúrico
}

\author{
Graciela V. Morales y Oscar D. Quiroga \\ Universidad Nacional de Salta, Facultad de Ingeniería, Consejo de Investigación, Instituto de \\ Investigaciones para la Industria Química, Avenida Bolivia 5150, 4400 Salta-Argentina \\ (e-mail: gmorales@unsa.edu.ar)
}

\begin{abstract}
Resumen
Se realizan estudios cinéticos de la disolución de hidroboracita en soluciones de ácido clorhídrico y ácido sulfúrico en un reactor tanque agitado discontinuo de acero inoxidable, a presión atmosférica. Se investiga el efecto de la temperatura, el tamaño de partícula, la relación sólido/líquido y la concentración de ácido sobre la velocidad de disolución. Los resultados experimentales permiten observar que la velocidad de disolución aumenta con el incremento de la concentración del ácido y de la temperatura, y disminuye con el aumento del tamaño de partícula y con la relación sólido/líquido. La energía de activación aparente calculada para la disolución de hidroboracita en soluciones de ácido clorhídrico es $44.9 \mathrm{~kJ} / \mathrm{mol}$, lo cual no permite inferir cual es la etapa controlante del proceso; mientras que la energía de activación aparente calculada para la disolución de hidroboracita en soluciones de ácido sulfúrico es $14.6 \mathrm{~kJ} / \mathrm{mol}$, lo cual permite inferir que el proceso ocurre con control difusivo.
\end{abstract}

Palabras claves: hidroboracita, ácido bórico, disolución, estudios cinéticos

\section{Dissolution Kinetics of Hydroboracite in Hydrochloric Acid and Sulphuric Acid Solutions}

\begin{abstract}
The dissolution kinetics of hydroboracite in hydrochloric acid and sulphuric acid solutions was investigated. The experiments were carried out in a steel batch reactor equipped with a mechanical stirrer, at atmospheric pressure. The effect of reaction temperature, particle size, solid/liquid ratio and acid concentration on the dissolution rate was determined. It was found that the dissolution rate increased with increasing the acid concentration and reaction temperature. However, the increase of particle size and solid/liquid ratio decreased the dissolution rate. The activation energy for the hydroboracite dissolution in hydrochloric acid was calculated as $44.9 \mathrm{~kJ} / \mathrm{mol}$, which does not permit to know the process controlling step. The activation energy for the hydroboracite dissolution in sulphuric acid was found to be $14.6 \mathrm{~kJ} / \mathrm{mol}$, which indicates that the dissolution is a diffusion controlled process.
\end{abstract}

Keywords: hydroboracite, boric acid, dissolution kinetics, kinetics studies 


\section{INTRODUCIÓN}

La hidroboracita es un borato de calcio y magnesio hexahidratado ( $\left.\mathrm{CaO} \cdot \mathrm{MgO} \cdot 3 \mathrm{~B}_{2} \mathrm{O}_{3} \cdot 6 \mathrm{H}_{2} \mathrm{O}\right)$. $\mathrm{La}$ provincia de Salta (Argentina) ocupa el primer lugar internacional en producción y exportación de hidroboracita, mineral que se explota en los yacimientos de la sierra de Sijes, en la Puna salteña (Alonso, 1986; Ortí y Alonso, 2000). El borato se presenta en cristales que forman agregados fibrosos y paralelos de brillo sedoso. Se caracteriza porque los agregados compactos son muy tenaces y los niveles mineralizados varían desde algunos milímetros hasta $2 \mathrm{~m}$ de espesor. La hidroboracita es insoluble en agua fría y caliente, pero se disuelve en agua acidificada. Sus usos están relacionados con la industria de los fertilizantes y otros compuestos para la agricultura, también se aplica en preparados de cerámicas especiales y como fundente en hornos metalúrgicos para la reducción de menas de titanio (Alonso, 1986).

Algunos investigadores han estudiado la disolución de boratos en soluciones ácidas. Imamutdinova (1967), estudió la disolución de ulexita en soluciones de $\mathrm{H}_{2} \mathrm{SO}_{4}, \mathrm{H}_{3} \mathrm{PO}_{4}, \mathrm{HNO}_{3}$ y $\mathrm{HCl}$. Demostró que en todos los casos el proceso de disolución es controlado por la difusión y que la velocidad de disolución es mayor en soluciones de $\mathrm{HNO}_{3}$ y menor en soluciones de $\mathrm{H}_{2} \mathrm{SO}_{4}$. En el caso de la disolución en soluciones de $\mathrm{H}_{2} \mathrm{SO}_{4}$ indicó que el proceso difusivo fue afectado negativamente por la formación de $\mathrm{CaSO}_{4}$ y $\mathrm{CaSO}_{4} \cdot 2 \mathrm{H}_{2} \mathrm{O}$ en adición al $\mathrm{H}_{3} \mathrm{BO}_{3}$. Por otra parte, Yapice et al. (1990) y Kocakerim et al. (1993), estudiaron la disolución de ulexita en soluciones acuosas de $\mathrm{CO}_{2}$. Ellos encontraron que el proceso de disolución es controlado por la difusión de $\mathrm{CO}_{2}$ en la solución. Künkül et al. (2003), estudiaron la disolución de ulexita en soluciones de sulfato de amonio y determinaron que, en este caso, la disolución es controlada también por mecanismos difusivos. Tunç et al. (1999), estudiaron la disolución de ulexita en $\mathrm{H}_{2} \mathrm{SO}_{4}$ y $\mathrm{HCl}$ y determinaron que la velocidad de disolución es mayor en soluciones de $\mathrm{HCl}$ que en soluciones $\mathrm{H}_{2} \mathrm{SO}_{4}$ debido a que, en este último caso tal como lo determinó Imamutdinova (1967), la película de producto que se forma sobre la superficie de mineral afecta la velocidad de disolución. Alkan et al. (2004), estudiaron los mecanismos cinéticos de la disolución de ulexita original y calcinada en soluciones de ácido oxálico y determinaron que la velocidad de disolución es controlada, también, por la difusión en la capa de producto; y Demirkiran y Künkül (2007), estudiaron la disolución de ulexita en soluciones de ácido perclórico. Por otra parte, Kurtbas et al. (2006), estudiaron la disolución de colemanita en soluciones acuosas de $\mathrm{SO}_{2}$ y ácido bórico. Ellos determinaron que la conversión del mineral puede ser bien correlacionada con el modelo de Avrami. Con respecto a la disolución de hidroboracita Morales et al. (2000), estudiaron la disolución de hidroboracita en agua con dióxido de carbono disuelto en soluciones diluidas (bajas relaciones sólido/líquido). Ellos observaron que el aumento de la temperatura favorece la velocidad de disolución del mineral, cualquiera sea el tamaño de la partícula, y que la velocidad de disolución se halla influenciada por fenómenos de transporte de masa de reactivos y/o productos.

En este trabajo se estudia la disolución de hidroboracita en soluciones diluidas de ácido clorhídrico $(\mathrm{HCl})$ y de ácido sulfúrico $\left(\mathrm{H}_{2} \mathrm{SO}_{4}\right)$. Se compara la velocidad de disolución del mineral en ambas soluciones ácidas y se investiga el efecto que tienen los principales factores (temperatura, tamaño de partícula, relación sólido/líquido y concentración de ácido) sobre la velocidad de disolución del mineral. Se evalúan los parámetros cinéticos: factor de frecuencia y energía de activación para la disolución de hidroboracita en las soluciones de $\mathrm{HCl}$ y $\mathrm{H}_{2} \mathrm{SO}_{4}$.

\section{METODOLOGIA}

Los experimentos de disolución de hidroboracita, se llevaron a cabo en un reactor de tipo tanque agitado discontinuo, de acero inoxidable, a escala laboratorio. La tapa del reactor posee: a) un orificio central por donde se introduce el eje de un agitador mecánico; b) un orificio descentralizado, que se utiliza para cargar la muestra de mineral y c) un tubo soldado que se utiliza para tomar las muestras de la fase líquida. Durante la experimentación el reactor se introdujo en un baño termostático con temperatura controlada y se mantuvo el medio agitado mecánicamente. Los experimentos fueron programados sobre la base de un diseño factorial a dos niveles, habiéndose seleccionado como factores (variables independientes) a los siguientes: $T$ (temperatura), $d_{p}$ (diámetro de partícula); Ra (relación masa de sólido a volumen de líquido) y $\mathrm{C}_{\mathrm{A}}$ (concentración del ácido). En la Tabla 1 se muestran los niveles de los factores usados en los experimentos. 
Tabla 1: Factores y sus valores usados en los experimentos

\begin{tabular}{|l|rr|}
\hline Tamaño de partícula $(\mathrm{mm}) ; \mathrm{d}_{\mathrm{p}}$ & 0,$326 ;$ & 0,774 \\
Temperatura $\left({ }^{\circ} \mathrm{C}\right): \mathrm{T}$ & $60 ;$ & 70 \\
Relación sólido/líquido $(\mathrm{g}(\mathrm{S}) / \mathrm{ml}(\mathrm{L})): \mathrm{Ra}$ & 0,$01 ;$ & 0,02 \\
Concentración del ácido $(\mathrm{N}): \mathrm{C}_{\mathrm{A}}$ & 0,$115 ;$ & 0,230 \\
\hline
\end{tabular}

Se mantuvo la velocidad de agitación constante e igual a 520 rpm después de verificar que la misma no tiene efectos significativos sobre la velocidad de disolución del mineral.

Se eligió como única respuesta del sistema al grado de transformación que experimenta el reactivo sólido con respecto de la masa de $\mathrm{B}_{2} \mathrm{O}_{3}$, y se la definió como:

$X=\frac{M_{B}^{\circ}-M_{B}}{M_{B}^{\circ}}$

donde $M_{B}^{\circ}$ es la masa inicial de $B_{2} O_{3}$ asociada a la partícula y $M_{B}$ es la masa asociada en un tiempo dado. Considerando que todos los experimentos se realizaron en un contactor de tipo tanque agitado discontinuo, entonces $X$ puede expresarse en función de la masa de $\mathrm{B}_{2} \mathrm{O}_{3}$ que se halla disuelta en la fase líquida:

$X=\frac{M_{B(L)}}{M_{B}^{o}}$

donde $\mathrm{M}_{\mathrm{B}(\mathrm{L})}=\mathrm{M}_{\mathrm{B}}^{\circ}-\mathrm{M}_{\mathrm{B}}$ es la masa de $\mathrm{B}_{2} \mathrm{O}_{3}$ que se halla disuelta en la solución en un tiempo dado.

La definición de $X$ dada por la ec. (2), fue precisamente la que se utilizó para seguir el curso de la disolución. Para ello, a tiempos de reacción previamente programados se tomaron muestras de la mezcla reaccionante, las cuales fueron inmediatamente filtradas. Del líquido filtrado se tomó una alícuota y se la diluyó con agua destilada. La solución diluida se analizó mediante volumetría.

\section{RESULTADOS Y DISCUSIÓN}

En la Fig. 1 se presentan los resultados experimentales de la disolución de hidroboracita en soluciones de $\mathrm{HCl}(0,23 \mathrm{~N})$ y $\mathrm{H}_{2} \mathrm{SO}_{4}(0,23 \mathrm{~N})$ a temperaturas de $60^{\circ} \mathrm{C}$ y $70{ }^{\circ} \mathrm{C}$, para un diámetro de partícula $\mathrm{d}_{\mathrm{p}}=0,774 \mathrm{~mm}$ y una relación sólido/ líquido, $\mathrm{Ra}=0,02 \mathrm{~g}(\mathrm{~S}) / \mathrm{ml}(\mathrm{L})$. Se observa que la velocidad de disolución del mineral es mayor en la solución de $\mathrm{HCl}$ para ambas temperaturas de disolución. Esta misma tendencia se presenta con el tamaño de partícula $d_{p}=0,326 \mathrm{~mm}$ y, para la relación sólido/líquido $\mathrm{Ra}=0,01 \mathrm{~g}(\mathrm{~S}) / \mathrm{ml}(\mathrm{L})$.

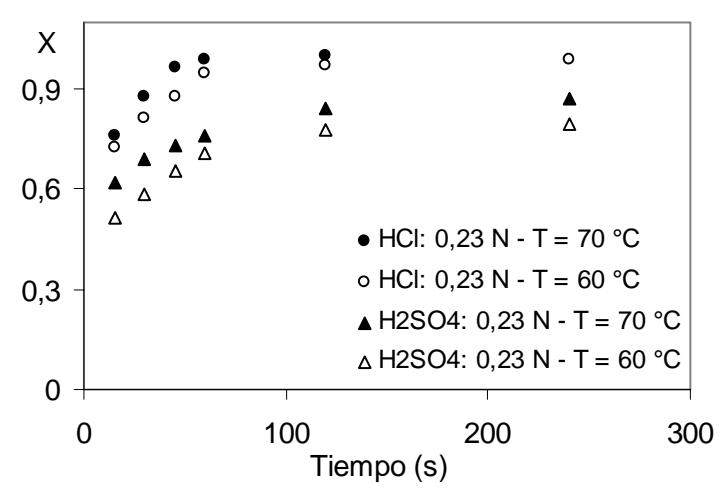

Fig. 1: Disolución de hidroboracita en $\mathrm{HCl}$ y $\mathrm{H}_{2} \mathrm{SO}_{4}$

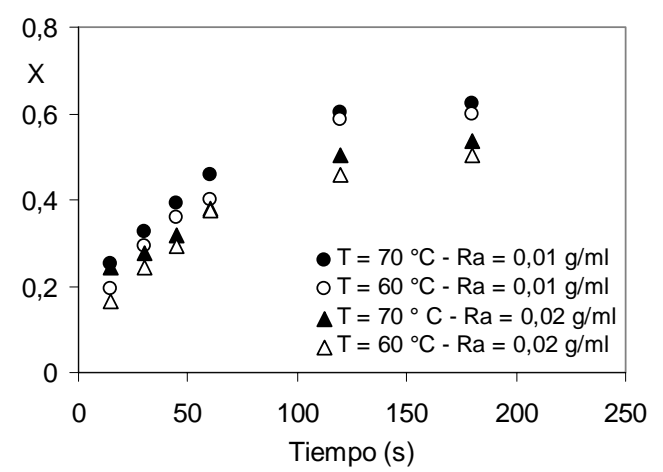

Fig. 2: Disolución en $\mathrm{HCl}$. Efecto de T y Ra

Por otra parte, en la Fig. 2 se presentan los resultados experimentales de la disolución de hidroboracita en $\mathrm{HCl}(0,115 \mathrm{~N})$ que se obtuvieron a $60{ }^{\circ} \mathrm{C}$ y $70{ }^{\circ} \mathrm{C}, \mathrm{d}_{\mathrm{p}}=0,774 \mathrm{~mm}$ y $\mathrm{Ra}=0,01$ 
$\mathrm{g}(\mathrm{S}) / \mathrm{ml}(\mathrm{L})$. Se presentan, también, los datos de la disolución de hidroboracita en $\mathrm{HCl}$ a iguales temperaturas, igual diámetro de partículas, igual concentración de ácido y a la relación sólido/líquido $\mathrm{Ra}=0,02 \mathrm{~g}(\mathrm{~S}) / \mathrm{ml}(\mathrm{L})$. Comparando entre sí cada una de las curvas de la Fig. 2, se observa que velocidad de la disolución aumenta con el incremento de la temperatura, y que a menor dilución, Ra $=0,01 \mathrm{~g}(\mathrm{~S}) / \mathrm{ml}(\mathrm{L})$, hay un mayor rendimiento de la transformación del mineral.

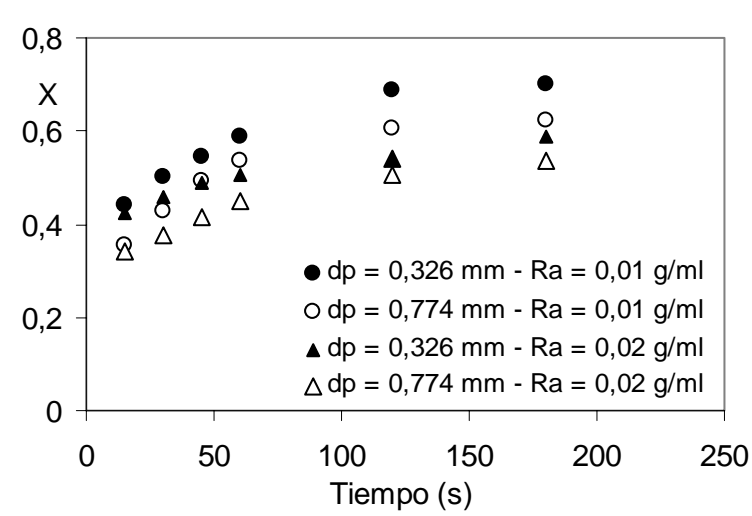

Fig. 3: Disolución en $\mathrm{HCl}$. Efecto de $d_{p}$ y Ra

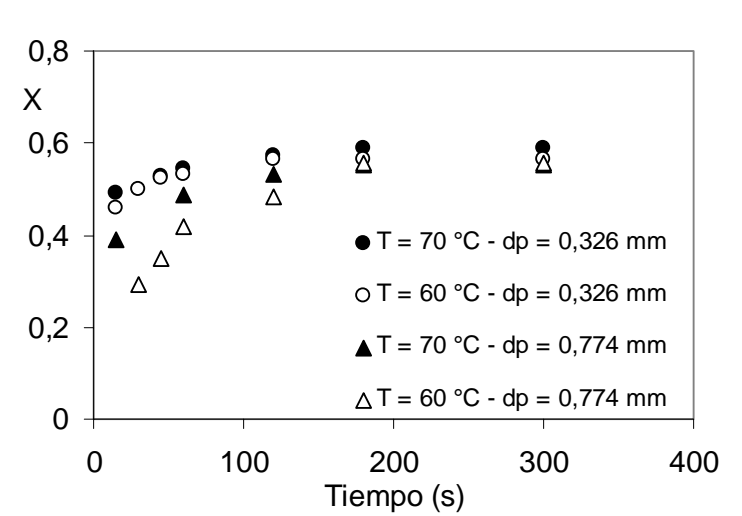

Fig. 4: Disolución en $\mathrm{H}_{2} \mathrm{SO}_{4}$. Efecto de $\mathrm{T}$ y $\mathrm{d}_{\mathrm{p}}$

En la Fig. 3 se presenta la influencia del tamaño de partícula sobre la disolución de hidroboracita en $\mathrm{HCl}(0,115 \mathrm{~N})$ a $70{ }^{\circ} \mathrm{C}$ y las relaciones sólido/líquido $\mathrm{Ra}=0,01 \mathrm{~g}(\mathrm{~S}) / \mathrm{ml}(\mathrm{L})$ y $\mathrm{Ra}=0,02 \mathrm{~g}(\mathrm{~S}) / \mathrm{ml}(\mathrm{L})$. Se observa,en ambos casos, que la velocidad de disolución aumenta con la disminución del tamaño de partícula del mineral. Este mismo efecto se manifiesta a $60^{\circ} \mathrm{C}$. Se puede observar, también en este caso, que a menor relación sólido/líquido hay un mayor rendimiento de la disolución del mineral.

Por otra parte, en la Fig. 4 se presentan los resultados experimentales de la disolución de hidroboracita en $\mathrm{H}_{2} \mathrm{SO}_{4}(0,115 \mathrm{~N})$ que se obtuvieron a $60{ }^{\circ} \mathrm{C}$ y $70{ }^{\circ} \mathrm{C}, \mathrm{d}_{\mathrm{p}}=0,326 \mathrm{~mm}$ y $\mathrm{Ra}=0,01$ $\mathrm{g}(\mathrm{S}) / \mathrm{ml}(\mathrm{L})$. Se presentan, también, los datos de la disolución de hidroboracita en $\mathrm{H}_{2} \mathrm{SO}_{4}(0,115 \mathrm{~N})$ que se obtuvieron a iguales temperaturas y un diámetro de partícula de $d_{p}=0,774 \mathrm{~mm}$. Se observa que la velocidad de disolución del mineral aumenta con el incremento de la temperatura y con la disminución del tamaño de partícula del mineral para ambas temperaturas.

Se realizaron, también, experimentos para investigar el efecto de la relación sólido/líquido en la disolución de hidroboracita en soluciones de $\mathrm{H}_{2} \mathrm{SO}_{4}$. Se observó que un aumento en la relación sólido/líquido hace disminuir el grado de transformación del mineral. Sin embargo, un incremento en la misma del doble hizo disminuir el grado de transformación del mineral únicamente en un $7 \%$.

\section{Cálculo de parámetros cinéticos}

En términos generales se sabe que la velocidad observada, $\mathbf{r}_{\mathrm{s}}^{\circ}$, de una transformación fluido-sólido reactivo dada, depende de numerosos factores como por ejemplo: la temperatura, $\mathrm{T}$, el diámetro de partícula, $d_{p}$, la concentración del reactivo fluido, $C_{A}$ y la relación sólido/líquido, $R a$. La influencia que tienen estos factores del sistema experimental sobre la velocidad inicial observada del proceso, puede resumirse cuantitativamente mediante la ecuación siguiente (Morales, 2002):

$$
\mathrm{r}_{\mathrm{s}}^{\mathrm{o}}=\mathrm{k}^{\mathrm{o}} \exp (-\mathrm{E} / \mathrm{RT}) \mathrm{C}_{\mathrm{A}}^{\mathrm{n}} \mathrm{d}_{\mathrm{p}}^{\mathrm{a}} \mathrm{Ra}^{\mathrm{b}}
$$

donde $\mathrm{k}^{0}$ es el coeficiente factor de frecuencia, E es el coeficiente energía de activación aparente y $\mathrm{n}$, a y b son coeficientes exponenciales. Estos coeficientes se estiman a partir de datos de la velocidad inicial calculados para cada uno de los niveles de los factores Si bien es cierto que la ec. (3) no es totalmente exacta, a través de las estimaciones de sus coeficientes es posible inferir cuál es la etapa controlante del proceso. Por ejemplo, para una reacción fluido-sólido reactivo, a través de los valores de la energía de activación, $\mathrm{E}$, puede inferirse si una reacción procede en régimen de control químico, difusivo ó mixto.

Para el cálculo de $\mathrm{r}_{\mathrm{S}}^{0}$ en función de los principales factores se ha seguido el procedimiento siguiente: 
i) Para un nivel dado de los factores, se correlacionan los datos X-t mediante el siguiente polinomio:

$X=b_{1} t+b_{2} t^{2}+b_{3} t^{3}+b_{4} t^{4}+\ldots$

donde t es el tiempo y los coeficientes $b_{1}, b_{2}$, etc., se estiman por regresión lineal.

ii) Se deriva la ec. (4) con respecto de t, se la evalúa en $t=0$ y se define la velocidad inicial de reacción:

$$
\left.\frac{\mathrm{dX}}{\mathrm{dt}}\right|_{\mathrm{t}=0}=\mathrm{r}_{\mathrm{S}}^{\mathrm{o}}=\mathrm{b}_{1}
$$

iii) Para el conjunto de experimentos realizados se formula $r_{S}^{0}$ como:

$$
\mathrm{b}_{1}=\mathrm{r}_{\mathrm{S}}^{\mathrm{o}}=\frac{\mathrm{k}^{\mathrm{o}} \exp (-\mathrm{E} / \mathrm{RT}) \mathrm{C}_{\mathrm{A}}^{\mathrm{n}}}{\mathrm{d}_{\mathrm{p}}^{\mathrm{a}} \mathrm{Ra}^{\mathrm{b}}}
$$

se estiman los coeficientes $k^{\circ}, E, n, a, b$.

\section{Caso 1) Disolución de hidroboracita en ácido clorhídrico}

Para procesar los datos experimentales se ha desarrollado un software específico, obteniéndose los siguientes resultados:

Velocidad inicial: $r_{S}^{o}=2,53 \times 10^{7} \exp \left(\frac{-5402}{T}\right) \frac{C_{A}^{1,072}}{d_{p}^{0,601} \mathrm{Ra}^{0,118}} \quad$ con $\quad E=44,9 \mathrm{~kJ} / \mathrm{mol}$

Se observa que: a) la velocidad inicial, $r_{S}^{o}$, es inversamente proporcional al tamaño de partícula, $d_{p}$, elevado a la potencia de 0,601. Este valor, menor que uno, permite inferir que la velocidad de disolución puede ser en algunos casos controlada por mecanismos difusivos y en otros por la velocidad de cambios estructurales internos de la partícula; b) la energía de activación aparente, E, es igual a 44,9 kJ/mol. Este valor corresponde al valor de una pseudo energía de activación que no permite, en este caso, inferir cual es la posible etapa controlante del proceso.

Caso 2) Disolución de hidroboracita en soluciones de ácido sulfúrico

Los datos experimentales fueron procesados por el software desarrollado, obteniéndose los siguientes resultados:

Velocidad inicial: $r_{S}^{o}=11,39 \exp \left(\frac{-1762}{T}\right) \frac{C_{A}^{0,840}}{d_{p}^{1,039} \mathrm{Ra}^{0,880}} \quad$ con $E=14,6 \mathrm{~kJ} / \mathrm{mol}$

Se observa que: a) la velocidad inicial, $r_{S}^{o}$, es inversamente proporcional al tamaño de partícula, $d_{p}$, elevado a la potencia de 1,039; b) la energía de activación aparente, E, es igual a 14,6 kJ/mol. Este valor permite inferir que en este caso la velocidad de disolución es controlada por efectos difusivos. Esto puede explicarse por el hecho de que la formación de iones sulfato, $\mathrm{SO}_{4}^{2-}$ da lugar a la precipitación de $\mathrm{CaSO}_{4}$ y $\mathrm{CaSO}_{4} .2 \mathrm{H}_{2} \mathrm{O}$ sólido sobre la superficie de la partícula (Tunç et al., 1999). Esta capa de producto sólido crea una dificultad para que los iones $\mathrm{H}_{3} \mathrm{O}^{+}$difundan al mineral, disminuyendo su velocidad de disolución.

\section{CONCLUSIONES}

Se han realizado experimentos de disolución de hidroboracita en soluciones de ácido clorhídrico y ácido sulfúrico. Todos ellos se llevaron a cabo con los siguientes factores en diferentes niveles: Ra, 
relación sólido/líquido; $T$, temperatura de disolución; $d_{p}$, diámetro de partícula; concentración del ácido, $\mathrm{C}_{\mathrm{A}}$ y velocidad de agitación, $\mathrm{n}_{\mathrm{r}}$. Se observa que el proceso de disolución en $\mathrm{HCl}$ es el que mas depende de la temperatura. Así para que la velocidad de disolución en $\mathrm{HCl}$ se duplique basta elevar la temperatura $15^{\circ} \mathrm{C}$, mientras que en medio ácido sulfúrico se debe elevar $50^{\circ} \mathrm{C}$.

En relación a la influencia de los factores $\mathrm{Ra}, \mathrm{d}_{\mathrm{p}}$ y $\mathrm{T}$ sobre la velocidad de disolución, se encontró que la misma aumenta con el aumento de la temperatura y la disminución del tamaño de partícula, y disminuye con el incremento de la relación sólido/líquido. Básicamente, esto concuerda con los aspectos teóricos generales de las reacciones líquido/sólido reactivo. Con respecto a la velocidad de agitación, estudios preliminares han mostrado que no tiene influencia sobre la velocidad de disolución. Los cálculos de los valores de las energías de activación aparente, E, permiten deducir lo siguiente: a) la disolución de hidroboracita en soluciones de ácido clorhídrico presenta una energía de activación aparente de $44,9 \mathrm{~kJ} / \mathrm{mol}$, que no permite inferir cual es la etapa controlante del proceso; b) el valor de la energía de activación aparente para la disolución de hidroboracita en soluciones de ácido sulfúrico $14,7 \mathrm{~kJ} / \mathrm{mol}$, permite inferir que el proceso ocurre con control difusivo.

\section{REFERENCIAS}

Alkan M., M. Dogan y H. Namli; Dissolution kinectis and mechanism of ulexite in oxalic acid solutions, Ind. Eng. Chem. Res.: 43, 1591-1598 (2004).

Alonso R.N.; Ocurrencia y posición estratigráfica y génesis de los depósitos de boratos de la Puna argentina, Tesis Doctoral, Facultad de Ciencias Naturales, Universidad Nacional de Salta: 1-196 (1986).

Imamutdinova, V. M.; Kinetics of dissolution of borates in mineral acid solutions, Zh. Prikl. Khim.: 40 (11), 2593-2596 (1967).

Demirkiran N. y A. Künkül; Dissolution Kinetics of ulexite in perchloric acid solutions, International Journal of Mineral Processing: 83, 76-80 (2007).

Kocakerim M.M., S. Çolak, T. Davies y M.Alkan; Dissolution kinetics of ulexite in $\mathrm{CO}_{2}$-Saturated water, J.Metall. Q: 32 (4), 393-396 (1993).

Künkül A., N. Demirkiran y A. Baysar; Dissolution kinetics of ulexite in ammonium sulfate solutions; Ind. Eng. Chem. Res.: 42, 982-986 (2003).

Kurbas A., M.M. Kocakerim, Ö. Küçük y A. Yartasi; Dissolution of colemanite in aqueous solutions saturated with both sulfur dioxide $\left(\mathrm{SO}_{2}\right)$ and boric acid, Ind. Eng. Chem Res.: 45, 1857-1862 (2006).

Morales G.V., M.E. Capretto, L. Mercado Fuentes y O.D. Quiroga; Dissolution kinetics of hydroboracite in water saturated with carbon dioxide. Hydrometallurgy: 58, 127-133 (2000).

Morales G.V.; Estudios básicos para el desarrollo de una nueva tecnología para producir ácido bórico en la Argentina, Tesis Doctoral, Facultad de Ingeniería, Universidad Nacional de Salta: 1- 246 (2002).

Ortí, F. y R.N. Alonso; Gypsum-Hydroboracite. Association in the Sijes Formation (Miocenom N W Argentine), Journal of Sedimentary Research: 70 (3), 664-681(2000).

Tunç M., Kocakerim, M. M., Yapici, S. e Y.S. Bayrakceken, Dissolution of ulexite in $\mathrm{H}_{2} \mathrm{SO}_{4}$ solution, Hydrometallurgy: 51, 359-370 (1999).

Yapice S., M.M. Kocakerim, A. Künkül; Optimization of production of $\mathrm{H}_{3} \mathrm{BO}_{3}$ from ulexite, Tr. J. Eng. Environ.Sci: 18,91-94 (1990). 